\title{
Competitive clustering in a bidisperse granular gas: Experiment, molecular dynamics, and flux model
}

\author{
René Mikkelsen, Devaraj van der Meer, Ko van der Weele, and Detlef Lohse \\ Department of Applied Physics and J. M. Burgers Center for Fluid Dynamics, University of Twente, P.O. Box 217, \\ 7500 AE Enschede, The Netherlands \\ (Received 11 July 2003; revised manuscript received 26 February 2004; published 20 December 2004)
}

\begin{abstract}
A compartmentalized bidisperse granular gas clusters competitively [R. Mikkelsen, D. van der Meer, K. van der Weele, and D. Lohse, Phys. Rev. Lett. 89, 214301 (2002)]: By tuning the shaking strength, the clustering can be directed either towards the compartment initially containing mainly small particles or to the compartment containing mainly large particles. Here, the conditions under which this competitive clustering occurs are studied experimentally, numerically (by means of molecular dynamics simulations), and analytically. A minimal model is derived that quantitatively accounts for the observed phenomena.
\end{abstract}

DOI: 10.1103/PhysRevE.70.061307

PACS number(s): 45.70.Mg, 45.70.Vn

\section{INTRODUCTION: EXPERIMENTAL OBSERVATIONS}

Clustering is one of the most characteristic features of granular gases. It not only makes them fundamentally different from ordinary molecular gases, but also plays a major role in many industrial applications where granular matter is sorted or transported [1-5]. The clustering arises from the inelastic collisions between the particles: If a region is slightly denser than the others, the particles collide more frequently and hence more energy is dissipated. This results in the formation of a cluster of slow moving particles. Vice versa, relatively dilute regions are depleted, with only a few rapid particles remaining. A particularly clear-cut view of the clustering process is obtained in a setup with two compartments, where vertical (not too strong) shaking spontaneously leads to one well-filled and one nearly empty compartment [6-8]. Similar clustering has also been found in a horizontally shaken system [9].

Until now, most attention has been given to clustering in monodisperse systems. In this paper we will focus on the clustering behavior of a bidisperse mixture of large and small particles. Recently, we have found that such a mixture clusters competitively [10]: By tuning the shaking strength, the clustering can be directed either towards the compartment initially containing mainly small particles, or to the one containing mainly large particles; see Fig. 1.

Our experimental setup consists of a cylindrical glass tube with inner diameter $11.2 \mathrm{~cm}$ and height $42.2 \mathrm{~cm}$, divided into two equal compartments by a wall of height $6 \mathrm{~cm}$. The tube is mounted on a shaker, which brings the system into a gaseous state through vertical, sinusoidal vibrations with adjustable frequency $f$ and amplitude $a$. In Fig. 1 we show two experimental runs, at different shaking strength, with a mixture of $P_{1}=300$ stainless steel beads of radius $r_{1}=2.50 \mathrm{~mm}$ and $P_{2}=600$ smaller ones of $r_{2}=1.25 \mathrm{~mm}$. The initial condition in both experiments is the same: $\{180$ large, 200 small $\}$ in the left compartment (A), and \{120 large, 400 small $\}$ in the right compartment (B). This means that in the initial situation $55 \%$ of the total particle mass is in compartment $\mathrm{A}$. Only the lower third part of the tube is shown in Fig. 1, since particles rarely go up all the way to the top lid.
For very strong shaking (not shown) the large and small particles distribute themselves uniformly over the two compartments. This will be denoted as regime 0 . In this case, the dissipation from the particle collisions is overwhelmed by the energy input into the system.

When we reduce the shaking strength below a certain threshold (see Sec. III for details), starting out from the same initial state, the particles form a cluster in compartment A; see Fig. 1, left column. This is regime I. The direction of the clustering is towards the larger total particle mass. It takes about half a minute for the cluster to develop.

For very mild shaking, however, the same initial condition surprisingly leads to a cluster in the other compartment: see Fig. 1, right column. We will call this regime II. The series of events is as follows: At first the large particles stay close to the floor, transferring energy from the vibrating bottom to the smaller ones above them, which thereby gain relatively high velocities. This is reminiscent of the demonstration experiment in which one puts a tennis ball on top of a basketball and lets them drop together: when they hit the ground, the tennis ball is "launched" and jumps much higher than its release height [11]. The effect is stronger in the left box (which has more large particles) than in the right box, and thus the small beads go preferentially into the latter (B). As a consequence, the remaining particles in compartment $\mathrm{A}$ become more mobile, and after a couple of minutes the first large beads also begin to make it over the wall into compartment B, where they are immediately swallowed by the developing cluster. With every particle that leaves compartment A, the process progressively speeds up. In the experiment of Fig. 1, right column, the clustering is complete after 15 minutes.

In the remainder of this paper we will give a quantitative description of this competitive clustering phenomenon in terms of a flux model and also through molecular dynamics (MD) simulations. Our goal is the construction of a minimal model that describes the clustering phenomena, rather than a general kinetic theory of the bidisperse gas. Specifically, we make three important simplifying assumptions regarding the energy equipartition in mixtures, the barometric height distribution, and the Maxwellian velocity distribution. Though 


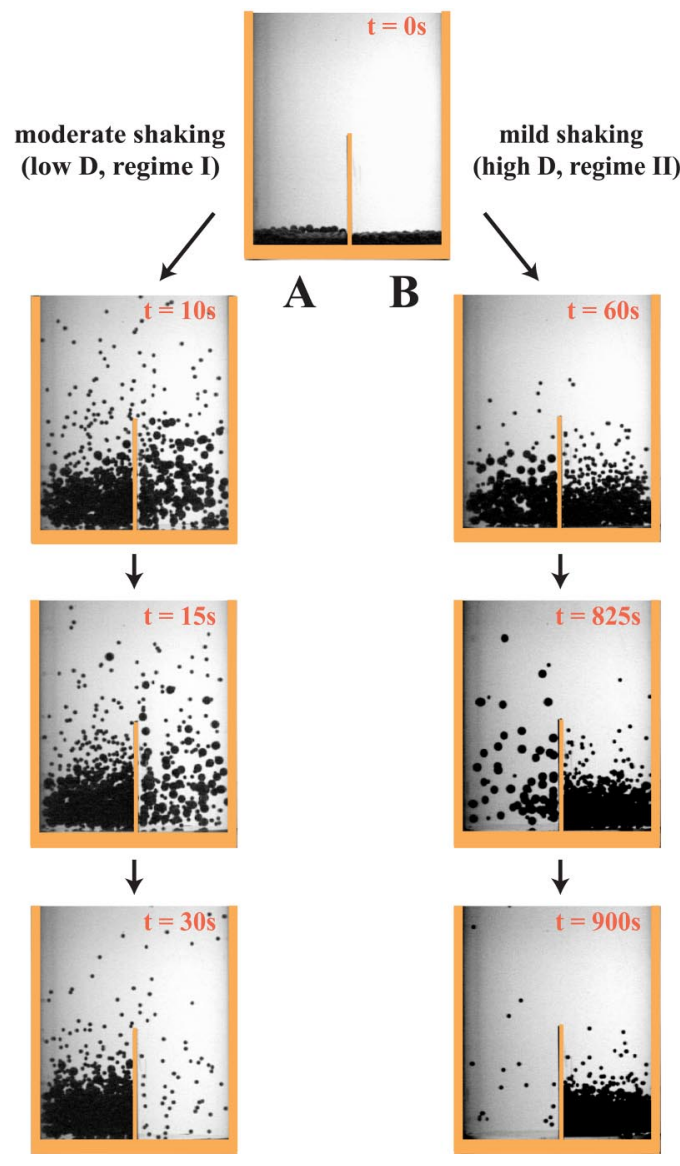

FIG. 1. Images from two experiments with a bidisperse mixture of steel beads, starting from the same initial condition. For relatively strong shaking (left column) the clustering is directed towards the left compartment, whereas for mild shaking (right column) it goes into the right compartment. The shaking frequency in the left column is $f=60.0 \mathrm{~Hz}$, and in the right column $37.5 \mathrm{~Hz}$, while the peak-to-peak amplitude in both cases is $2 a=2 \mathrm{~mm}$. The initial condition (topmost picture) is $\{180$ large, 200 small $\}$ in the left compartment, and $\{120$ large, 400 small $\}$ in the right one. With the radius of the large beads $\left(r_{1}=2.50 \mathrm{~mm}\right)$ being twice that of the small ones $\left(r_{2}=1.25 \mathrm{~mm}\right)$, this means that initially $55 \%$ of the total particle mass is in the left compartment.

it may appear that our minimal model bypasses the state of the art in granular matter, it is definitely sufficient to account for the experimental and numerical results on competitive clustering.

Throughout the paper we will work with particles of the same material, which thus have the same material density $\rho$ and will be taken to have one constant coefficient of restitution $\epsilon$; that is, we neglect the dependence of $\epsilon$ on the velocity and size of the particles $[12,13]$. The number of particles is taken such that at rest they form one to two layers on the bottom of the container. This number is sufficiently large to keep the relative effect of statistical fluctuations limited, and thus to allow for a mean field description. On the other hand, it is small enough to keep the gas reasonably dilute even in the clustered situation. The clusters in Fig. 1 look very dense, but this is an optical illusion due to the fact that the particles are projected onto a plane; in reality they are scattered throughout the three-dimensional compartment.

In Sec. II, MD simulations are employed to check two of the main approximations used in the flux model, namely, that the particles in each compartment obey a barometric height distribution, and that the small and large particles have roughly the same granular temperature. In Sec. III the flux model is worked out. In the main text we emphasize the physical ideas, while the mathematical derivation is given in an Appendix.

In Sec. IV we show, on the basis of the flux model, that the transition from regime I to II is directly related to a shift of the boundary between the basins of attraction associated with the two different clustered situations. We also study the dependence of the clustering behavior on the size ratio $\psi$ $=r_{1} / r_{2}$ and the total particle numbers $P_{1}$ and $P_{2}$. Predictions from the flux model are put side-by-side with measurements obtained from MD simulations and laboratory experiments, and fair agreement is found. Finally, Sec. V contains concluding remarks.

\section{MD SIMULATIONS FOR ONE COMPARTMENT}

\section{A. Numerical scheme}

For the simulations we use a three-dimensional event driven code: Between two events (collisions) the particles move freely, describing parabolic paths under the influence of gravity, until the next collision occurs. A collision can be either between particles or between a particle and a wall, and is signaled by a spatial overlap of the two. At such an event, the velocities of the particles after contact are computed from the velocities just before contact using Newton's laws.

The particles are taken to be hard spheres. This means that we ignore any deformations, which for the steel particles used in our experiments is a reasonable approximation. The coefficient of normal restitution $\epsilon$ for particle-particle collisions is taken to be constant, $\epsilon=0.85$, and the same for the large and small beads. The coefficients of tangential restitution and dynamical friction are adjustable in the code, but for the simulations presented here they are set equal to their ideal (dissipationless) values.

The coefficients of restitution between the particles and the walls and bottom can be adjusted independently. For the coefficient of normal restitution we use 0.95 , obtained from test experiments in which we let the beads bounce on solid plates of glass (representing the walls) and aluminum (for the bottom).

For simplicity, the experimental setup is simulated as a rectangular box with infinitely high side walls. The ground area of each compartment, and also the height of the wall between them is the same as in the actual experiment. The bottom is vibrated vertically with adjustable frequency $f$ and amplitude $a$ following a sinusoidal wave form.

\section{B. Height distribution and granular temperature}

One of the main assumptions in the bidisperse flux model (see Sec. III) is that the granular temperatures $T_{1}$ and $T_{2}$ of the large and small particles are independent of the height $z$. This assumption leads to the barometric height distribution, 

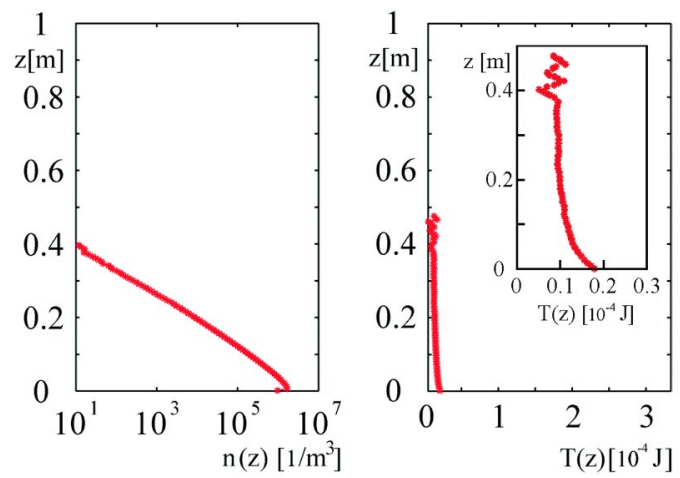

FIG. 2. Molecular dynamics (MD) simulation results for a monodisperse granular gas (size ratio $\psi=1$ ) shaken at frequency $f$ $=70 \mathrm{~Hz}$ and amplitude $a=1 \mathrm{~mm}$. Shown are the particle number density (left) and the granular temperature (right) as function of the height $z$. The ground area of the container is $\Omega=100 \mathrm{~cm}^{2}$ (equivalent to the experimental setup of Fig. 1 without the central wall), the number of particles is $P=900$, their radius is $1.25 \mathrm{~mm}$, and the plots are based on $10^{6}$ numerical snapshots of the gas in its steady state, sampled at a rate of 1000 per second. The scale used in these plots is the same as in Figs. 3 and 4, to allow for a comparison with the bidisperse case.

just as in an ordinary gas. That is, the number densities of both species $(i=1,2)$ are taken to decay exponentially with $z$ :

$$
n_{i}(z)=n_{i}(0) e^{-m_{i} g z / T_{i}} .
$$

The temperature $T_{i}$ is defined in analogy with the standard relation from statistical physics $\frac{1}{2} m_{i}\left\langle v_{i}^{2}\right\rangle=\frac{3}{2} k_{B} T_{i}$, where the Boltzmann constant $k_{B}$ is to be replaced by a mere number. Here we will choose $k_{B}=1$. So $T_{i} \equiv \frac{1}{3} m_{i}\left\langle v_{i}^{2}\right\rangle$, directly proportional to the mean kinetic energy of the particles of species $i$. Its value is determined by a balance between the input of energy due to the vibrating bottom and the dissipation of energy via the collisions [14].

The second, bolder assumption in the model is that $T_{1}$ $=T_{2}(=T)$. The large and small particles are (per compartment) taken to be in thermal equilibrium with each other at the same granular temperature, and Eq. (1) then simplifies to $n_{i}(z)=n_{i}(0) \exp \left\{-m_{i} g z / T\right\}$. For size ratios $\psi$ close to unity (the monodisperse limit) this is expected to hold well, but for large values of $\psi$ the correspondence will deteriorate. Several recent studies [15-20] have shown that energy equipartition generally breaks down in bidisperse granular gases, with the heavier particles having a higher temperature.

In particular, Wildman and Parker [15] used positron emission particle tracking to experimentally determine the granular temperature in a vibrofluidized mixture of glass beads with radii $r_{1}=2.5 \mathrm{~mm}$ and $r_{2}=2.0 \mathrm{~mm}(\psi=1.25)$. They found that the temperature of the larger particles was always higher than that of the smaller ones. Keeping the total particle mass in the system the same, the temperature ratio $T_{1} / T_{2}$ could be raised by increasing the ratio $\left(P_{1} / P_{2}\right)$ between the numbers of large and small beads.

To check the temperature ratio and the density profiles in our own system, we performed MD simulations. In Figs. 2-4 the results are shown for $\psi=1,2$, and 3 , respectively.
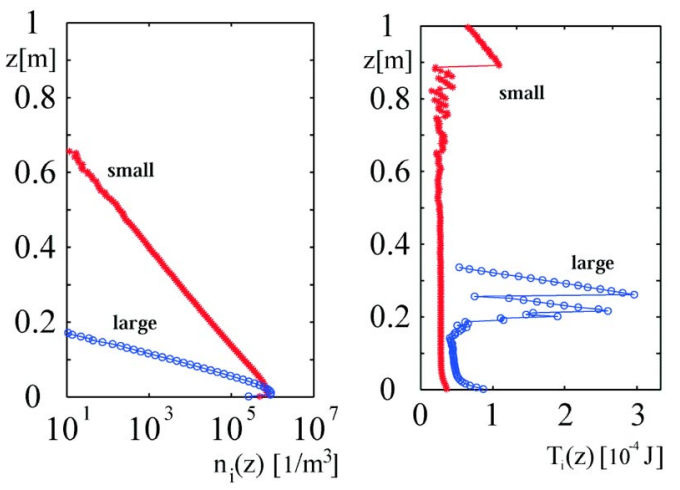

FIG. 3. Same as Fig. 2, but now for a bidisperse granular gas consisting of 300 large and 600 small particles with size ratio $\psi$ $=2$. The small particles have the same size as those in Fig. 2, i.e., $r_{2}=1.25 \mathrm{~mm}$. The density profiles of both the large and the small particles follow straight lines, indicating an exponential decay with $z$ (barometric height distribution). The right plot shows that the granular temperature of the large beads is larger than that of the small beads. The slanting lines at the top of the temperature profiles correspond to free parabolic flights of single particles; see also Fig. 5.

In all three cases $\log \left[n_{i}(z)\right]$ (for $i=1$ and 2) follows an approximately straight line, indicating that the density profiles of both large and small beads indeed decay exponentially, with the large-particle profile decaying faster, in agreement with Eq. (1). Only at small $z$ the profiles deviate significantly from the straight line. This is caused by the vibrating floor: Many of the particles here have a relatively high energy, since they have just been kicked by the floor but have had no chance yet to pass on their energy to the other particles. So the temperature close to the bottom is high and this means that the curve of $\log \left(n_{i}(z)\right)$ flattens. Moreover, a very narrow region immediately above the floor is swept clean by the vibrating bottom itself.
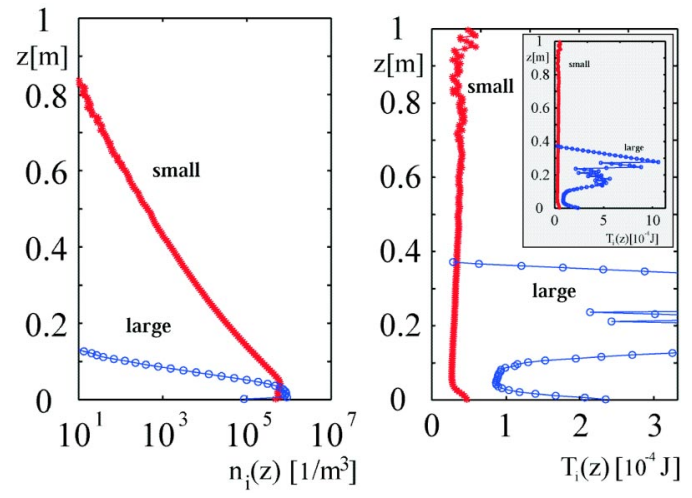

FIG. 4. Same as Fig. 3, but now for size ratio $\psi=3$. The densities of the large and small particles still follow an approximately exponential decay. The temperature shows considerable deviations from a constant value, especially for the large particles (see also the inset, in which the same profiles are shown on a different scale); however, the upper region of the temperature profile is made up by only a few particles and has hardly any statistical weight. It is apparent that the temperature difference between the large and small particles has increased with $\psi$; cf. Fig. 6 . 

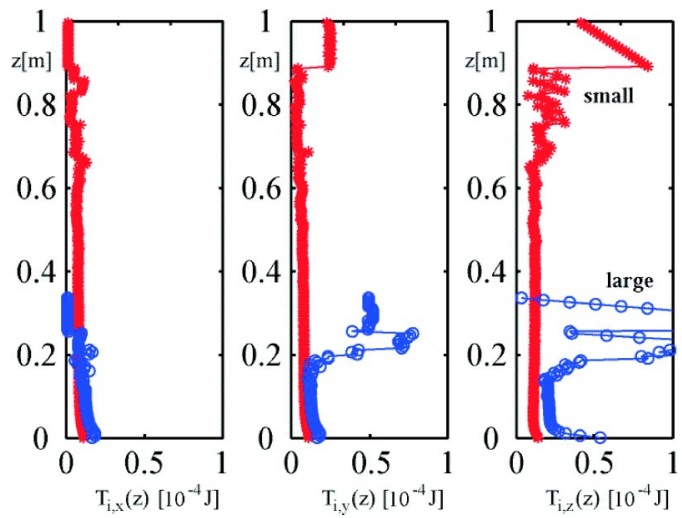

FIG. 5. The $x, y$ and $z$ components of the granular temperature for the bidisperse mixture of Fig. 3, $\psi=2$. These temperature components are directly proportional to the kinetic energies of the particles: $T_{i, x}=\frac{1}{3} m_{i} v_{i, x}^{2}$, etc. $(i=1,2$ denoting the large and small particles, respectively).

An interesting observation is that the small particles in the bidisperse situations (Figs. 3 and 4) reach considerably larger heights, and have a higher temperature, than the same particles in the monodisperse situation of Fig. 2. This is the "tennis ball on basketball" effect mentioned in the Introduction. The maximum height reached by the small particles increases with $\psi$, i.e., with the growing size of the larger particles.

The above characteristics are also reflected in the temperature profiles. The temperature is found to be roughly constant except at the bottom and top. Close to the bottom the temperature is significantly higher, especially for the large particles. That this is indeed caused by the vertical kicks from the vibrating floor is shown in Fig. 5, where the individual $x, y$, and $z$ components of the temperature are given (for $\psi=2$ ): For the large particles, the $z$ component close to the bottom is seen to be almost three times as high as the other two components.

In the bulk of the profile (the long central part) the temperature components are roughly equal, which means that the velocity distribution is approximately constant and isotropic here.

The upper part of the temperature profile shows considerable fluctuations. The reason for this is that the particle density is rather low here, so (a) the statistics is relatively poor and (b) the collisions between particles are rare, which makes the equipartition of energy via collisions less effective. In this region the mean free path of the particles increases rapidly with height and their kinetic energy is primarily converted into potential energy due to gravity and not lost in collisions.

In the uppermost region (above $z=0.9 \mathrm{~m}$ in Fig. 5) we see the ballistic behavior of an individual small particle that freely travels upward, reaches the top (velocity in the $z$ direction becomes zero at $z=1.10 \mathrm{~m}$, outside the plot), and goes down again. The velocity components in the $x$ and $y$ direction remain practically constant during this parabolic flight [21]. A ballistic regime is also apparent in the temperature profiles of the larger particles: two parabolic flights that go considerably higher than the rest of the large particles,

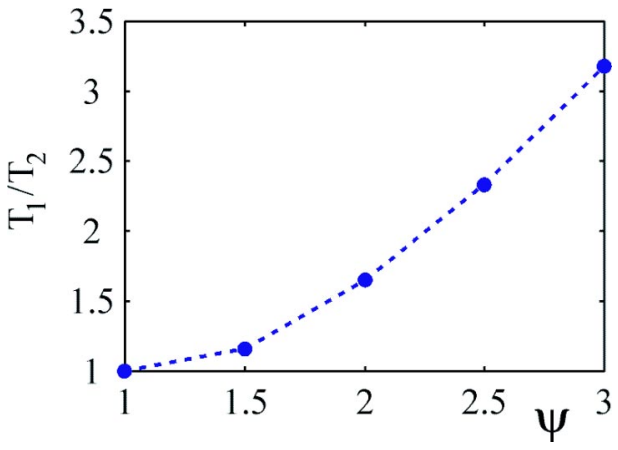

FIG. 6. Temperature ratio $T_{1} / T_{2}$, determined from MD simulations, as function of the size ratio $\psi=r_{1} / r_{2}$. The values are taken at $z=0.075 \mathrm{~m}$, which lies in the "constant" part of the large-particle temperature profile for each $\psi$.

and are not thwarted by the surrounding small ones, are clearly visible in Fig. 3 (and Fig. 5) around $z=0.22-0.35 \mathrm{~m}$. Indeed, the pronounced increase of the large-particle temperature toward the top of their range is due to the fact that the large particles observed at these heights are the ones that have chanced to fly up straight from the bottom, with no (or very few) collisions on the way up. The increase of the temperature profile here thus roughly reflects the temperature peak at the bottom.

The simulations show that the large particles have a higher temperature than the small ones, in agreement with the results found in the recent literature on this subject [15-20]. In Fig. 6 the temperature ratio $T_{1} / T_{2}$ as estimated from our MD simulations, is given as a function of the size ratio $\psi=r_{1} / r_{2}$. The values in this plot hold at a height where the temperature profiles of both species are approximately constant; in the present case we have chosen $z=0.075 \mathrm{~m}$ for each value of $\psi$. They can be read off directly from the temperature profiles (see Figs. 2-4), or indirectly from the density profiles, by using the following relation between the slopes [from the barometric height equation (1)] :

$$
\frac{\text { slope large-particle profile }}{\text { slope small-particle profile }}=\frac{m_{1} T_{2}}{m_{2} T_{1}}=\psi^{3} \frac{T_{2}}{T_{1}} .
$$

Both methods yield the same value for the temperature ratio.

In summary, we find that both species are not in equilibrium with the same granular temperature unless their mass ratio is one. On the other hand, even for $\psi=2$, where the corresponding mass ratio $m_{1} / m_{2}$ is 8 , the temperature ratio is still less than 1.7. Here the assumption of energy equipartition (with $T_{1} / T_{2}=1$ ) is still a meaningful first approximation.

\section{FLUX MODEL}

\section{A. Basic equations and approximations}

The flux model describes the flow of large and small particles between the compartments, as a function of the particle numbers in each compartment and of the shaking strength. It is a bidisperse generalization of Eggers' model for a monodisperse granular gas [7]. For its derivation we first consider the gas in a single compartment, and from its steady state 
behavior determine the so-called flux function, i.e., the number of particles of each species that leaves the compartment per unit time [22]. In order to keep the model transparent, we make three main approximative assumptions that are highlighted below.

Barometric height distribution. The particles in each compartment are taken to obey the following equation of state [23]:

$$
p_{i}=n_{i} k_{B} T_{i}
$$

(the ideal gas law, with $k_{B}=1$ in the present context) and the momentum balance

$$
\frac{d p_{i}}{d z}=-m_{i} g n_{i}
$$

for both species $i=1,2$ separately. Combining these two equations under the assumption that the granular temperature $T_{i}=\left(m_{i} / 3 k_{B}\right)\left\langle v_{i}\right\rangle^{2}$ is independent of $z$ gives $k_{B} T_{i} d n_{i} / d z$ $=-m_{i} g n_{i}$. Integration gives the barometric height formula:

$$
n_{i}(z)=n_{i}(0) e^{-m_{i} g z k_{B} T_{i}} .
$$

In the previous section we saw that this exponential distribution describes the real situation remarkably well, given the fact that both the ideal gas law (3) and the assumption that $T_{i}$ is independent of $z$ only hold in an approximate sense. One might make the agreement even better by using a more refined equation of state [24-26] and by letting $T$ vary with $z$ [27], but this would make an analytical expression for the flux function very difficult (if not impossible) while not affecting the resulting height distribution too much.

Energy equipartition. The assumption that both species have the same granular temperature $\left(T_{i}=T\right.$ for $\left.i=1,2\right)$ strictly speaking means that we confine ourselves to size ratios $\psi$ close to 1; see Fig. 6. Nevertheless, also for $\psi=2$ and even for $\psi=3$ the model turns out to give results that closely agree with our experiments and MD simulations. This implies that the inequality of $T_{1}$ and $T_{2}$ does not play an essential role in the competitive clustering effect, and the assumption $T_{1} / T_{2}$ $=1$ may thus be viewed as an application of Occam's razor in order to keep the theory as simple as possible. In the Appendix we will indicate how the model can be extended to a temperature ratio $T_{1} / T_{2}$ different from 1 .

The density at ground level in Eq. (5) follows from the condition $\Omega \int_{0}^{\infty} n_{i}(z) d z=N_{i}$ :

$$
n_{i}(0)=\frac{m_{i} g N_{i}}{\Omega k_{B} T},
$$

where $N_{i}$ is the number of particles (of species $i$ ) in the compartment under consideration and $\Omega$ is its ground area.

The temperature $T$ should be interpreted as an average value for the whole compartment. Its value is determined by balancing the energy input via the vibrating bottom and the energy loss through the interparticle collisions (both per unit of time):

$$
J_{0}=\Omega \int_{0}^{\infty} q(z) d z
$$

Here $J_{0}$ is the energy input rate, and $q(z)$ is the dissipation rate per volume. For the sake of simplicity we neglect the energy loss resulting from collisions with the wall, i.e., we treat those collisions as being completely elastic.

Maxwellian velocity distribution. The third important simplification is that we assume the velocity distribution of the particles to be Maxwellian and isotropic. This is an approximation, both with respect to the Maxwellian nature [28,29] as to the isotropy (see Fig. 5), allowing us to calculate both sides of Eq. (7) in closed form. This is done in the Appendix and we arrive at the following expression for the granular temperature $T$ of the compartment:

$$
k_{B} T=\frac{(2 a f)^{2} \mu}{16 \pi\left(1-\epsilon^{2}\right)^{2}}
$$

where the effective mass $\mu$ is given by

$$
\mu\left(N_{1}, N_{2}\right)=\left(\frac{\Omega\left(m_{1} N_{1}+m_{2} N_{2}\right)}{r_{1}^{2} \sqrt{m_{1}} N_{1}^{2}+r_{2}^{2} \sqrt{m_{2}} N_{2}^{2}+\left(r_{1}+r_{2}\right)^{2} \sqrt{\frac{1}{2} m_{12}} N_{1} N_{2}}\right)^{2},
$$

with $m_{12}=m_{1} m_{2} /\left(m_{1}+m_{2}\right)$. It is through this quantity $\mu$ that the particle numbers of the two species enter the temperature. One may check that in the monodisperse limit $\left(r_{1}=r_{2}\right.$ $\left.=r, m_{1}=m_{2}=m\right)$ it reduces to $\mu_{\psi=1}=\Omega^{2} m /\left[r^{2}\left(N_{1}+N_{2}\right)\right]^{2}$ $=m\{\pi \Omega / \text { total projected area of the particles }\}^{2}$, i.e., the particle mass divided by the square of a dimensionless filling factor.

The temperature from Eq. (8) compares well with the temperatures $T_{1}$ and $T_{2}$ of the large and small particles in the MD simulations of Figs. 2-4. Indeed, for $\psi=2,3$ one finds the temperature from Eq. (8) to be in between $T_{1}$ and $T_{2}$. It is slightly larger (about 10\%) than the weighted average of $T_{1}$ and $T_{2}$, as can be understood from the idealizations in the model. For example, the model does not take into account the dissipation from the particle-wall collisions, and assumes a sawtooth driving instead of the sinusoidal driving used in the simulations.

\section{B. Flux function}

The central quantity of the model is the flux function $F_{i}$, defined as the number of particles (of species $i$ ) that leaves the compartment per unit time. It is the product of half the density $\frac{1}{2} n_{i}(z)$ (so that we count particles moving in one direction only) and the average horizontal velocity (which is equal to $\sqrt{2 k_{B} T / \pi m_{i}}$ ) integrated over the space above the wall (width $b$ ) from $z=h$ to some cutoff height $h+H$. Above the cutoff height, the state variables of the two compartments are in equilibrium and hence no net flux occurs. In principle, $H$ will depend on the mean free path of the particles, but here we take it to be constant. The integration is then straightforward [30]: 

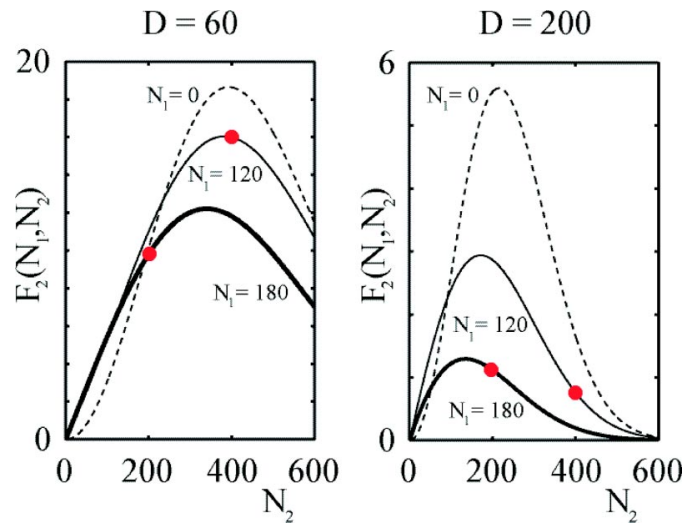

FIG. 7. The small-particle flux $F_{2}\left(N_{1}, N_{2}\right)$ as a function of $N_{2}$, for various numbers of large particles in the compartment: $N_{1}=0$ (dashed), $N_{1}=120$ (thin), and $N_{1}=180$ (thick). For $D=60$ (relatively strong shaking; left plot) the flux from a compartment with $\{180$ large, 200 small $\}$ particles (indicated by the left dot) is smaller than from a compartment with $\{120$ large, 400 small $\}$ particles (right dot). Hence the clustering is towards the former compartment, i.e., type I clustering, in agreement with the experimental observation of Fig. 1. For $D=200$ (weak shaking; right plot) it is the other way around, leading to type II clustering, again in agreement with experiment. The "tennis ball on basketball effect" is most pronounced on the left flank of the flux function, where the small-particle flux from the compartment actually rises (with respect to the dashed curve) upon adding large particles. Note the different scales of the vertical axis in the two plots: the stronger the shaking, the higher the particle flux.

$$
\begin{aligned}
F_{i}\left(N_{1}, N_{2}\right) & =\frac{1}{2} \sqrt{\frac{2 k_{B} T}{\pi m_{i}}} b \int_{h}^{h+H} n_{i}(z) d z \\
& =\sqrt{\frac{k_{B} T}{2 \pi m_{i}}} \frac{b N_{i}}{\Omega} e^{-m_{i} g h / k_{B} T}\left(1-e^{-m_{i} g H / k_{B} T}\right) \\
& \approx K N_{i} \sqrt{\frac{m_{i}}{\mu}} e^{-D m_{i} / \mu}, \quad i=1,2 .
\end{aligned}
$$

In the last step we have linearized $\exp \left(-m_{i} g H / k_{B} T\right)$, implying that $H \ll\left\langle v_{i}^{2}\right\rangle / g$, and expressed $k_{B} T$ in terms of the particle numbers $N_{i}$ by means of Eqs. (8) and (9). The prefactor $K$ determining the absolute rate of the flux is given by [31]

$$
K=2 \sqrt{2}\left(1-\epsilon^{2}\right) \frac{g b H}{\Omega(2 a f)},
$$

and the dimensionless parameter $D$, which governs the clustering behavior, has the form

$$
D=16 \pi \frac{g h}{(2 a f)^{2}}\left(1-\epsilon^{2}\right)^{2} .
$$

The influence of the large particles on the small-particle flux (and vice versa) is contained in the parameter $\mu$, given by Eq. (9).

In Fig. 7 we show the small-particle flux $F_{2}\left(N_{1}, N_{2}\right)$ as a function of $N_{2}$, at $D=60$ (relatively strong shaking) and $D$ $=200$ (weak shaking) respectively, for three different values of the number of large particles $N_{1}$.

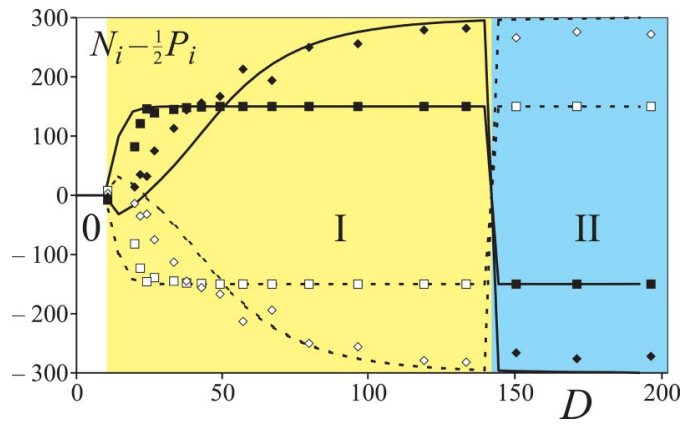

FIG. 8. Bifurcation diagram showing the three different clustering regimes $\mathrm{O}, \mathrm{I}$, and II. The particle numbers $N_{i}(i=1,2)$ are given relative to the symmetric solution: $N_{i}-\frac{1}{2} P_{i}$. The curves represent the steady state according to the theoretical flux model and the squares and diamonds are experimental data. For the experiments we used the setup shown in Fig. 1, filled with a mixture of stainless steel beads: $P_{1}=300$ large ones with radius $r_{1}=2.5 \mathrm{~mm}$, and $P_{2}$ $=600$ small ones with radius $r_{2}=1.25 \mathrm{~mm}$. Every new run was started from the same initial condition: $\{180$ large, 200 small $\}$ in compartment A, and $\{120$ large, 400 small $\}$ in B. The squares correspond to the large beads, and the diamonds to the small ones. Solid symbols refer to compartment A, and open symbols to B; note that every measurement is thus represented by two points, which accounts for the mirror-symmetry of the plot in the vertical direction.

For $N_{1}=0$ (no large particles in the compartment, dashed curve) the flux function has the well-known monodisperse form studied in Refs. [7,8,32,33]. It starts out from zero at $N_{2}=0$ (expressing the fact that there is no particle flux from an empty compartment) and initially increases with growing $N_{2}$. For any ordinary molecular gas it would always keep increasing, but for a granular gas it is seen to reach a maximum and goes down again: The inelastic collisions (which become more and more frequent as $N_{2}$ grows) make the particles slow, until they are hardly able to jump over the wall anymore and the flux $F_{2}\left(0, N_{2}\right)$ approaches zero in the limit for $N_{2} \rightarrow \infty$.

For $N_{1}=120$ (thin curve) and $N_{1}=180$ (thick curve) the maximum of the flux function decreases as compared to the situation without large particles (dashed curve), due to the much larger total mass in the compartment. However, on the left flank there is a region where the drawn curves are actually higher than the dashed one. This is an illustration of the "tennis ball on basketball effect" mentioned in the Introduction, with the small particles becoming more mobile thanks to the presence of the larger ones.

One can deduce the type of clustering that results from the plots in Fig. 7. Let us start, just as in the experiments of Fig. 1, with $\{180$ large, 200 small $\}$ particles in one compartment (A) and $\{120$ large, 400 small $\}$ particles in the other (B). In the left plot, for $D=60$, we see that the flux from compartment A (indicated by the left dot) is smaller than from compartment $\mathrm{B}$ (right dot). Hence the direction of clustering is towards A, i.e., type I clustering, in agreement with the experimental observation of Fig. 1 (see also Fig. 8). In the plot for $D=200$ it is precisely the other way around, resulting in type II clustering, again in agreement with experiment. Note also the different scales along the vertical axis in the two 
plots: The flux function is considerably smaller for weak shaking, confirming the fact that the clustering process takes much longer there.

With the above flux function we are now in a position to calculate the dynamics of our two-compartment system, starting from any initial condition, and for any shaking strength. The evolution of the number of particles $N_{i A}$ in compartment $\mathrm{A}(i=1,2)$ is given by the net balance between the (outgoing) flux from A to B and the (incoming) flux from B to A:

$$
\begin{aligned}
\frac{d N_{i A}}{d t} & =-F_{i}\left(N_{1 A}, N_{2 A}\right)+F_{i}\left(N_{1 B}, N_{2 B}\right) \\
& =-F_{i}\left(N_{1 A}, N_{2 A}\right)+F_{i}\left(P_{1}-N_{1 A}, P_{2}-N_{2 A}\right),
\end{aligned}
$$

where we have used particle conservation, $N_{i A}+N_{i B}=P_{i}$. The evolution of the (complementary) particle numbers in compartment $\mathrm{B}$ is governed by the same equation with $\mathrm{A}$ and $\mathrm{B}$ interchanged.

\section{COMPARING THE FLUX MODEL, EXPERIMENT, AND MD SIMULATIONS}

\section{A. Competitive clustering for size ratio $\psi=2$}

The predictions from the flux model, calculated from Eqs. (10)-(13), are found to be in good quantitative agreement with our experimental results. In Fig. 8 we compare model predictions and experimental data for a mixture of $P_{1}=300$ large and $P_{2}=600$ small steel beads, with size ratio $\psi=2$, starting always from the same initial situation:

$$
\begin{aligned}
& \left\{N_{1 A}(0), N_{2 A}(0)\right\}=\{180,200\} \text { in compartment A, } \\
& \left\{N_{1 B}(0), N_{2 B}(0)\right\}=\{120,400\} \text { in compartment B. }
\end{aligned}
$$

Both in the model and in experiment we recover the three different regimes observed in the Introduction: For vigorous shaking (regime $\mathrm{O}, D<10$ ) the system quickly settles into a symmetric state with equal amounts of small and large particles in both compartments. At moderate shaking (regime I, $10<D<140$ ) the clustering takes place in compartment A, the one initially containing the majority of large particles. This regime has been indicated by a light (yellow) shading in Fig. 8 and in all figures that follow. At even milder shaking (regime II, $D>140$ ) the clustering takes place in compartment $\mathrm{B}$; for this regime we use a darker shading (blue).

The time scale of the clustering grows with increasing $D$. This is illustrated in Fig. 9, where the evolving particle numbers $N_{i A}(t)$ and $N_{i B}(t)$ (evaluated by the flux model) are given at $D=100$ and 200, respectively. In the first case, the clustering is complete already after $150 \mathrm{~s}$, whereas in the latter case it takes almost a hundred times as long. In agreement with our experimental observations, the small particles cluster first, and only when nearly all of them have reached their final destination the large ones follow. The clustering times obtained from the flux model are in reasonable agreement with the experimental observations, including a sudden jump in the time scale at the transition from type-I to type-II clustering: Just before the transition the clustering (into box A) is
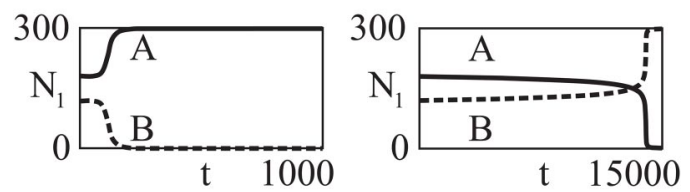

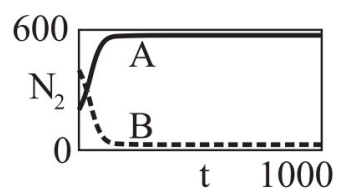

(a)

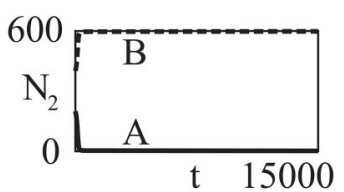

(b)
FIG. 9. Evolution of the system calculated from the flux model, starting from the initial condition Eq. (14), for (a) $D=100$ (type-I clustering) and (b) $D=200$ (type-II clustering). The solid curves represent the number of particles in compartment $\mathrm{A}$; the dashed ones compartment B. It is seen that the small particles (lower row) cluster first, followed by the large ones (top row). Note the different time scales between type-I and type-II clustering.

experimentally found to be about 10 times as fast as just after the transition (into box B). This jump is also found in the time scales evaluated from the flux model.

In order to see what causes the transition from regime I to II we make flow diagrams (see Fig. 10) that show how the particle numbers $N_{1 B}(t)$ and $N_{2 B}(t)$ in compartment B evolve, for any initial condition. The arrows indicate the dynamics of the system, and the cross denotes the initial condition that was used in the experiments [specified in Eq. (14)].

For very strong shaking [Fig. 10(a), $D=1$ ] only one fixed point exists: the stable uniform distribution $\{150,300\}$ in the center of the flow diagram. The system quickly approaches this point regardless of the initial condition.

At $D=20$, just beyond the pitchfork bifurcation, the homogeneous state has become unstable and has given way to two new stable fixed points. These correspond to compartment $\mathrm{B}$ being either comparatively empty (fixed point in the lower part of the flow diagram, type I clustering) or well filled (upper part, type II). The basins of attraction for these two points are indicated by the shading: Any initial condition in the light (yellow) region will lead to a cluster in box A, while initial conditions lying in the dark (blue) region lead to a cluster in box B. The initial condition for the experiments of Fig. 8 (indicated by the cross) lies in the yellow basin, so this one leads to a cluster in compartment A. The arrows indicate that first the small particles settle into their preferred distribution over the compartments, and that the large ones follow later (as we also noted in the plots of Fig. 9). At this relatively small value of $D$ the small beads are still divided over the two compartments, but the large beads already cluster heavily: This is in agreement with the bifurcation diagram of Fig. 8.

For $D=80$ the clustering has become much more pronounced, since also the small beads accumulate into the same compartment. We furthermore note that the boundary between the two basins of attraction has shifted and is now almost horizontal.

At very mild shaking $(D=200)$, the boundary between the two basins of attraction has shifted again. The initial condition (the cross) now lies within the blue basin of attraction, 


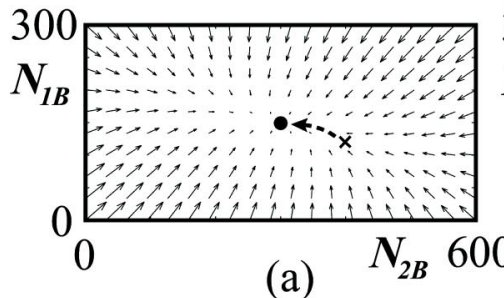

(a)

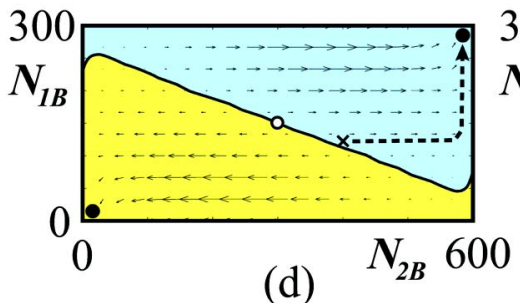

(d)

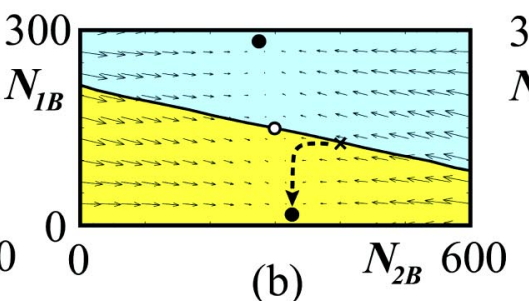

(b)

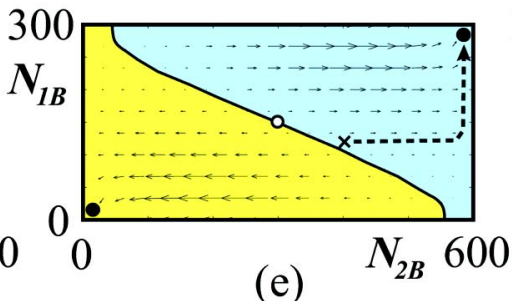

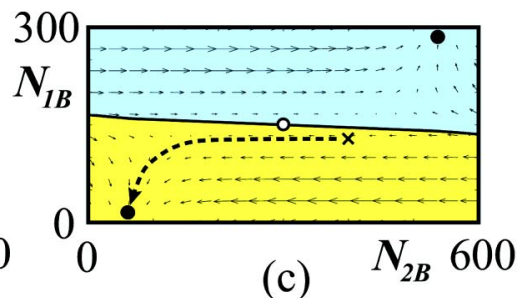

(c)

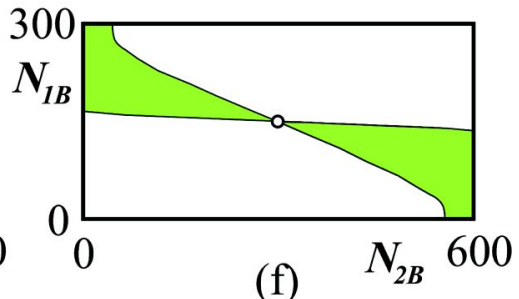

FIG. 10. Flow diagrams calculated from the flux model (for the same system as in Figs. 8 and 9) showing how the contents of compartment B evolve at five successive values of the shaking parameter: (a) $D=1$, (b) $D=20$, (c) $D=80$, (d) $D=200$, and (e) $D=250$. The cross indicates the initial condition used in the experiments: $\left\{N_{1 B}(0), N_{2 B}(0)\right\}=\{120,400\}$. At $D=1$ there is no clustering and all initial conditions lead to the uniform distribution (the central point in the flow diagram). For $D=20, \ldots, 250$ all initial conditions in the light (yellow) basin of attraction lead to a comparatively empty compartment B (type-I clustering) and those in the dark (blue) basin of attraction lead to a well-filled compartment B (type-II clustering). Note that the slope of the boundary between the two regimes shows nonmonotonic behavior as a function of $D$. Part (f) shows the region of competitive clustering: The boundary between the two basins of attraction in (a)-(e) sweeps through this region, and therefore the initial conditions here lead to either type-I or type-II clustering depending on the value of $D$.

and we end up with nearly all particles in compartment B. The same plot shows that the fixed points move further into their corners for increasing $D$, i.e., the clustering becomes more pronounced for decreasing shaking strength. This feature was apparent already in Fig. 1, and has been observed earlier also for clustering in a monodisperse gas, i.e., for $\psi$ $=1[7,8]$.

Interestingly, the boundary between the two basins of attraction is found to move (as function of $D$ ) in a nonmonotonic fashion. From $D=20$ to $D=80$ it is seen to straighten out towards an almost horizontal position, but from $D=80$ onwards it starts to slant again and at the same time develops a curve. At some point between $D=200$ and $D=250$ it goes through the diagonal position and eventually seems to come to a standstill. We shall not pursue the limit for very high values of $D$, however, since here the shaking becomes so weak that no particles are able to jump over the wall anymore: Any clustering predictions in this limit will no longer be reproducible in experiments or MD simulations.

The motion of the basin boundary shows that competitive clustering does not occur for all initial situations: Only a set of conditions in the lower right quadrant and (equivalently) the upper left quadrant can be directed into either compartment by tuning the shaking strength. On the other hand, there is also a region through which the boundary sweeps twice, so here we find two consecutive transitions between the clustering regimes I and II as $D$ is varied. The initial condition used in the experiment lies just outside this double transition region; had it been chosen slightly differently, the bifurcation diagram of Fig. 8 would have had an additional band of type-II clustering between regimes $\mathrm{O}$ and $\mathrm{I}$. The twist in the small-particle curve immediately after the pitchfork bifurcation at $D=20$ is a "ghost" of this band.

\section{B. Exploring the parameter space: \\ Dependence on size ratio $(\psi)$ and relative abundance of large and small particles $(\sigma)$}

\section{Size ratio $\psi$}

How do the above observations generalize to size ratios $\psi=r_{1} / r_{2}$ differing from 2 ? This ratio has a marked effect on the critical $D$ values where the transition from the regimes $\mathrm{O}$, I, and II take place. In Figs. 11 and 12 we show the position of these regimes as a function of $\psi$ and $D$, for the same initial condition that was specified in Eq. (14). The drawn curves have been calculated from the flux model, and the symbols

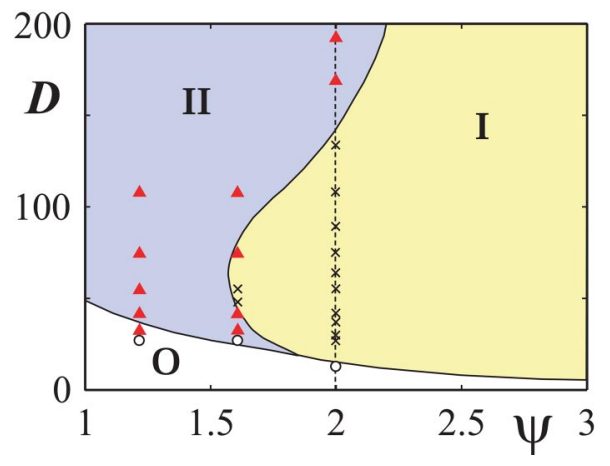

FIG. 11. Phase diagram, showing the three clustering regimes as a function of the inverse shaking strength $D$ and the size ratio $\psi$ $=r_{1} / r_{2}$. The drawn curves are calculated from the flux model, and the symbols correspond to experiments: open circles $=$ no clustering, crosses=type-I clustering, and red triangles=type-II clustering. The experimental results on the vertical dashed line $\psi$ $=2$ also feature in Fig.8. The initial condition is always taken to be as in Eq. (14). 


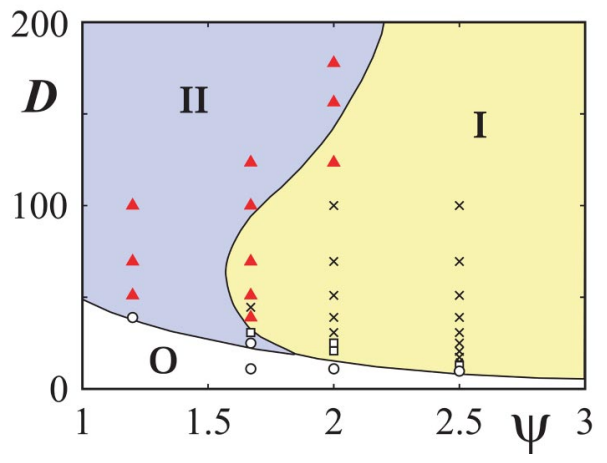

FIG. 12. The same phase diagram as in Fig. 11, for the same initial situation (14), but this time the symbols represent MD simulations. Between the regimes of type-I and type-II clustering, there is also a zone where the clustering in the MD simulations can go either way, depending on statistical fluctuations. This undecided state of affairs is indicated by the open squares: Each of them is based on 10 repetitions of the MD simulation, of which typically half ended in type-I clustering and the other half in type-II. As in all previous figures, the ratio of large to small particles is $\sigma=P_{1} / P_{2}$ $=300 / 600=1 / 2$.

are data from experiments (Fig. 11) and MD simulations (Fig. 12). The vertical dashed line in Fig. 11 corresponds to the case $\psi=2$ studied in the previous subsection.

It is seen that for $\psi<1.5$ the transition from regime $\mathrm{O}$ to regime II is immediate: here the larger beads are not sufficiently big to compensate for the fact that they are a minority. It is the larger number of beads that decides where the cluster goes, just as for the monodisperse case $(\psi=1)$. On the other hand, for high values of $\psi$, the dominant size of the large beads always makes them the decisive factor (only regime I survives). It is precisely the intermediate region 1.5 $<\psi \lesssim 2.3$ in which the competition takes place: The curving border between regimes I and II indicates the critical value of $D$ where the basin boundary sweeps through our initial condition (14). For $\psi \approx 1.6$ the boundary sweeps twice through this initial condition and we witness the particularly interesting sequence 0 -II-I-II, both in the model and in experiment.

Both in experiment (Fig. 11) and in the MD simulations (Fig. 12) the actual border between regimes I and II is found to lie more to the right than predicted by the flux model. This shift of the borderline means that the "counterintuitive" type-II clustering is even stronger than predicted by the flux model. This may be understood from the fact that the mobility of the large beads is underestimated by the flux model, which assumes the granular temperatures for the large and the small beads to be equal. In reality (in experiments and MD simulations) the temperature of the large ones is known to be higher, and therefore the type-II scenario in which the majority of large beads switches compartment occurs somewhat easier than suggested by the flux model.

\section{Relative abundance $\sigma$}

In the experiments and simulations so far we have always used mixtures in which the number of large particles was half the number of small ones: $\sigma=P_{1} / P_{2}=1 / 2$. Let us now have a brief look at other compositions, since obviously this
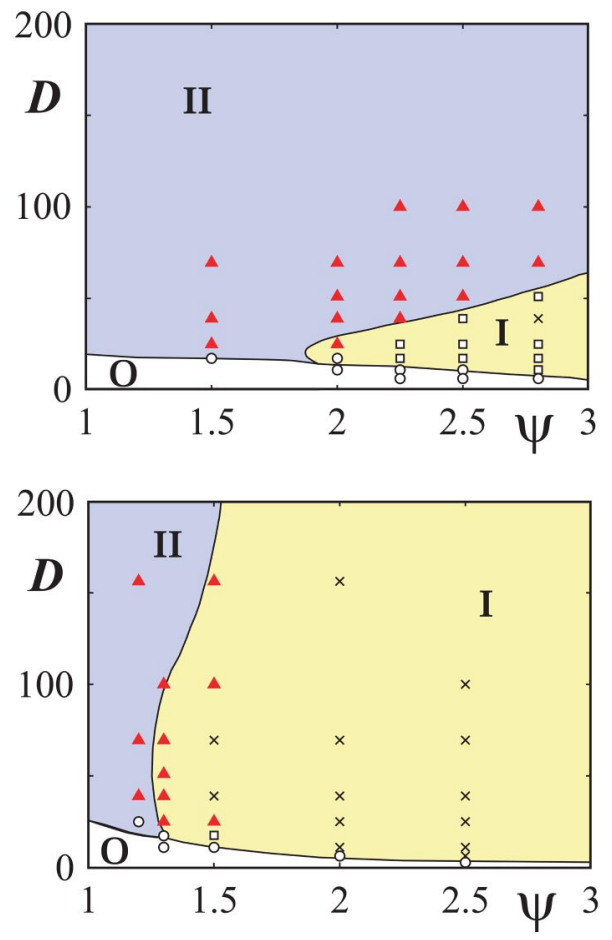

FIG. 13. The same as Fig. 12, but now for (a) $\sigma=P_{1} / P_{2}$ $=200 / 1200=1 / 6$ and (b) $\sigma=600 / 600=1$. For growing $\sigma$ (relative abundance of the large particles) type-I clustering clearly gains ground. The initial condition used here is $\left\{\frac{3}{5} P_{1}, \frac{1}{3} P_{2}\right\}$ in compartment $\mathrm{A}$ and (hence) $\left\{\frac{2}{5} P_{1}, \frac{2}{3} P_{2}\right\}$ in compartment $\mathrm{B}$, in analogy with the condition (14) which was taken in all previous figures (where $\sigma=300 / 600=1 / 2$ ).

parameter $\sigma$ must have an important influence on the clustering behavior: A larger value of $\sigma$ means that the large beads become a more important minority (or even a majority for $\sigma>1$ ), and hence type-I clustering will gain ground. This is indeed the case, as illustrated by Fig. 13 for $\sigma=1 / 6$ and 1 . The initial condition we use here $\left(\left\{\frac{3}{5} P_{1}, \frac{1}{3} P_{2}\right\}\right.$ in compartment $\mathrm{A}$ and $\left\{\frac{2}{5} P_{1}, \frac{2}{3} P_{2}\right\}$ in compartment $\mathrm{B}$ ) is equivalent to the one taken in all previous experiments and simulations [Eq. (14)], but due to the change in $P_{1}$ and $P_{2}$, the absolute number of particles initially inserted into the two compartments are different.

The position of all the lines (i.e., transitions) in the phase diagram are affected by the changing particle numbers. Take, e.g., the value of $D$ at which the transition from regime $\mathrm{O}$ to II occurs in the monodisperse limit $\psi=1$. This clearly goes down as the total number of particles in the system increases: In Fig. 12 (with $P_{1}+P_{2}=900$ ) the critical $D$-value exceeds 40, while in Fig. 13(a) (with $P_{1}+P_{2}=1400$ ) it lies below 20. The physical reason for this is that the larger number of particles induces more collisions, and hence the dissipation rate increases, so stronger shaking is necessary to obtain the homogeneous distribution. According to the monodisperse flux model $[7,8,32]$ the critical $D$-value for $\psi=1$ goes as

$$
D_{c, \psi=1} \propto \frac{1}{\left(P_{1}+P_{2}\right)^{2}} .
$$

That is, the product of $D_{c, \psi=1}$ and $\left(P_{1}+P_{2}\right)^{2}$ is exactly the same in all three plots of Figs. 12, 13(a), and 13(b). 
The border between the two clustered states (regime I and II) is affected even more drastically. Particularly the band of $\psi$ values where both clustering types can be obtained by adjusting the shaking strength (competitive clustering) depends strongly on $\sigma$. For $\sigma=1$ it is confined to the narrow band of values $1.2<\psi<1.5$. Here regime I dominates the phase diagram and the borderline between type-I and type-II clustering is pushed towards the vertical axis at $\psi=1$.

For decreasing $\sigma$ the same borderline moves towards the right and bends down, thereby reducing regime I and broadening the band of competitive clustering. Indeed, in Fig. 13(a) for $\sigma=1 / 6$, there is only one point (indicated by the cross) which consistently gave type-I clustering in our MD simulations. It is surrounded by a number of points for which the clustering was undecided, sometimes going in one direction and sometimes in the other; this is a manifestation of statistical fluctuations, which are not taken into account in our mean field approach [34]. Not surprisingly, given the relatively large values of $\psi$ in this region (and the associated deterioration of the one-temperature assumption, see Fig. 6) the simulations do not precisely follow the predictions of the flux model here. Nevertheless, the general trend of the phase diagram is still well reproduced.

\section{CONCLUSION}

In conclusion, a simplified phenomenological flux model quantitatively and consistently captures the physics of the competitive clustering phenomenon in a bidisperse granular gas: In the model, just as in experiment and MD simulations, the clustering can be directed either towards the compartment initially containing the majority of large particles (type-I clustering) or to the one containing mainly small particles (type-II), simply by adjusting the shaking strength.

The best quantitative agreement between the numerical simulations and the theoretical model is found when the size ratio between the large and small particles $(\psi)$ is not too much larger than 1 . This can be traced back to the fact that in the model the granular temperatures $T_{1}$ and $T_{2}$ are assumed to be equal, which is an accurate assumption only for $\psi$ close to 1 . Since the region of competitive clustering is found to move closer and closer towards $\psi=1$ if we let the number of large particles grow (see Fig. 13), this means that the theory works best for comparatively large numbers of large particles.

For smaller large-particle numbers the region of competitive clustering in the phase diagram is pushed towards higher $\psi$ values. The theoretical description here becomes less accurate, but still shows the correct qualitative features. Our MD simulations show that in these regions the borderline between type-I and type-II clustering widens to a broad zone where the cluster can go in either direction.

\section{ACKNOWLEDGMENTS}

We want to thank Peter Reimann for fruitful discussions about the theoretical model. This work is part of the research program of FOM, which is financially supported by the
Dutch Science Organization NWO; RM and DvdM acknowledge financial support.

\section{APPENDIX: DERIVATION OF THE FLUX MODEL}

In this Appendix we calculate respectively the left- and right-hand side of Eq. (7), i.e., the energy input into a compartment via the vibrating bottom $\left(J_{0}\right)$ and the energy loss through the particle-particle collisions $\left[\Omega \int_{0}^{\infty} q(z) d z\right]$, both per unit of time. Equating these two quantities leads to the granular temperature $T$ of the compartment, given by Eq. (8).

\section{Energy input}

The energy input comes from collisions of the particles with the bottom. For simplicity, we assume a sawtooth motion of the bottom, such that colliding particles always find it moving upwards with velocity $v_{b}=2 a f$. The peak-to-peak amplitude $2 a$ is taken to be sufficiently small compared to the mean free path of the particles, so that the bottom is effectively stationary.

Thus, when a particle with downward vertical velocity component $v_{z i}$ collides with the bottom, it is reflected back with an upward vertical velocity of $v_{z i}+2 v_{b}$. The energy gain per collision is equal to the difference in kinetic energy before and after collision, i.e.,

$$
\Delta E_{k i n}=2 m_{i} v_{b}\left(v_{z i}+v_{b}\right) .
$$

To obtain the total energy input rate, this expression must be multiplied by the number of collisions per unit time, which is $\frac{1}{2} n_{i}(0)\left|v_{z i}\right| \Omega$ for each species (with the factor $\frac{1}{2}$ representing the fact that half of the particles have a downward vertical velocity component), and averaged over all possible $v_{z i}$. Now, let us assume that the velocity distribution is Maxwellian and isotropic. As already mentioned in the main text, this is an approximation both with respect to the Maxwellian nature $[28,29]$ as to the isotropy (see Fig. 5) to keep the model as simple as possible. It allows us to set $\left\langle v_{z i}^{2}\right\rangle=\frac{1}{3}\left\langle v_{i}^{2}\right\rangle$ $=k_{B} T / m_{i}$ and $\left\langle\left|v_{z i}\right|\right\rangle=\sqrt{2 k_{B} T / \pi m_{i}}$, yielding the following expression for the rate of energy input:

$$
J_{0}=\Omega \sum_{i=1,2} n_{i}(0)\left(v_{b} k_{B} T+\sqrt{\frac{2}{\pi}} v_{b}^{2} \sqrt{m_{i} k_{B} T}\right) .
$$

This equation can easily be generalized to two different temperatures $T_{i}$ for the species $i=1,2$, but we will not do so here. Since the velocity of the bottom $v_{b}$ is typically much smaller than the velocity of the particles $\left(v_{b} \ll v_{i}\right)$, the first term in Eq. (A2) is much larger than the second, which we therefore neglect. The energy input then becomes

$$
J_{0}=\Omega v_{b} k_{B} T\left(n_{1}(0)+n_{2}(0)\right)=g v_{b}\left(m_{1} N_{1}+m_{2} N_{2}\right),
$$

where in the last step we have used Eq. (6).

\section{Energy loss}

To evaluate $q(z)$, the dissipation rate per volume, we consider a particle of mass $m_{i}$ and velocity $\mathbf{v}_{\mathbf{i}}$ traveling through a 
bidisperse background. If it collides with another particle of mass $m_{j}$ and $\mathbf{v}_{j}$ the energy loss will be, on the average (i.e., averaged over the collisional cross section):

$$
E_{\text {loss }}=\frac{1}{4} \frac{m_{i} m_{j}}{m_{i}+m_{j}}\left(1-\epsilon^{2}\right)\left(\mathbf{v}_{i}-\mathbf{v}_{j}\right)^{2},
$$

which happens to be precisely half of the energy loss in a frontal collision.

A collision will happen (within a time interval $d t$ ) if either of the two particles finds itself in the cross-sectional volume of the other, which is a cylinder of length $\left|\mathbf{v}_{i}-\mathbf{v}_{j}\right| d t$ and front area $\pi\left(r_{i}+r_{j}\right)^{2}$. Hence the collision rate per volume is the product of the particle densities $n_{i}(z) n_{j}(z)$ and this crosssectional volume divided by $d t$, where we assume that the densities do not vary significantly over this volume.

The dissipation rate per volume $[q(z)]$ is found by multiplying the collision rate per volume with the energy loss (A4), and averaging over all possible realizations of the independently distributed velocities $\mathbf{v}_{i}$ and $\mathbf{v}_{j}$ :

$$
q(z)=\frac{1}{8}\left(1-\epsilon^{2}\right) \sum_{i, j=1}^{2} n_{i}(z) n_{j}(z) \pi\left(r_{i}+r_{j}\right)^{2} \frac{m_{i} m_{j}}{m_{i}+m_{j}}\left\langle\left|\mathbf{v}_{i}-\mathbf{v}_{j}\right|^{3}\right\rangle,
$$

where we have multiplied by an additional factor $\frac{1}{2}$ to balance the fact that in this procedure we count every collision twice.

To evaluate the ensemble average $\left\langle\left|\mathbf{v}_{i}-\mathbf{v}_{j}\right|^{3}\right\rangle$, we note that-under the assumption of Maxwellian velocity distributions-all of the components of $\mathbf{v}_{i}$ and $\mathbf{v}_{j}$ are distributed Gaussian with variances (i.e., squared standard deviations) $\sigma_{i}^{2}=k_{B} T / m_{i}$ and similarly for $\sigma_{j}^{2}$. Again, it is possible to generalize this to two different temperatures $T_{i}$ for the species $i=1,2$, but here we will continue to work with $T_{1}$ $=T_{2}=T$.

It follows that the components of the combined variable $\mathbf{u}_{i j} \equiv\left(\mathbf{v}_{i}-\mathbf{v}_{j}\right) / \sqrt{2}$ are also Gaussian, with zero mean, and its variances are found by adding those of the (independently distributed) constituents $\mathbf{v}_{i}$ and $\mathbf{v}_{j}$ and dividing by 2: $\sigma^{2}$ $=k_{B} T / 2 m_{i}+k_{B} T / 2 m_{j}$. The distribution function $P\left(\mathbf{u}_{i j}\right)$ thus equals

$$
\begin{aligned}
P\left(\mathbf{u}_{i j}\right) & =\frac{1}{\left(2 \pi \sigma^{2}\right)^{3 / 2}} e^{-u_{i j}^{2} / 2 \sigma^{2}} \\
& =\left(\frac{m_{i} m_{j}}{\left(m_{i}+m_{j}\right) \pi k_{B} T}\right)^{3 / 2} \exp \left\{\frac{-m_{i} m_{j} u_{i j}^{2}}{\left(m_{i}+m_{j}\right) k_{B} T}\right\},
\end{aligned}
$$

where $u_{i j}=\left|\mathbf{u}_{i j}\right|$, and with this we can calculate

$$
\begin{aligned}
\left\langle\left|\mathbf{v}_{i}-\mathbf{v}_{j}\right|^{3}\right\rangle & =2 \sqrt{2}\left\langle\left|\mathbf{u}_{i j}\right|{ }^{3}\right\rangle=8 \pi \sqrt{2} \int_{0}^{\infty} u_{i j}^{5} P\left(u_{i j}\right) d u_{i j} \\
& =\frac{16}{\sqrt{2 \pi}}\left(\frac{k_{B} T\left(m_{i}+m_{j}\right)}{m_{i} m_{j}}\right)^{3 / 2} .
\end{aligned}
$$

Inserting this expression in Eq. (A5) we find

$$
\begin{aligned}
q(z) & =\sqrt{2 \pi}\left(1-\epsilon^{2}\right)\left(k_{B} T\right)^{3 / 2} \sum_{i, j=1}^{2} n_{i} n_{j}\left(r_{i}+r_{j}\right)^{2}\left(\frac{m_{i}+m_{j}}{m_{i} m_{j}}\right)^{1 / 2} \\
& =8 \sqrt{\pi}\left(1-\epsilon^{2}\right)\left(k_{B} T\right)^{3 / 2}\left(\frac{n_{1}^{2} r_{1}^{2}}{\sqrt{m_{1}}}+\frac{n_{2}^{2} r_{2}^{2}}{\sqrt{m_{2}}}+\frac{n_{1} n_{2}\left(r_{1}+r_{2}\right)^{2}}{2 \sqrt{2} \sqrt{m_{12}}}\right),
\end{aligned}
$$

with $m_{12}=m_{1} m_{2} /\left(m_{1}+m_{2}\right)$ the so-called reduced mass. The energy dissipation rate $Q$ now follows by integrating $q(z)$ over the whole volume of the compartment:

$$
\begin{aligned}
Q= & \Omega \int q(z) d z \\
= & \sqrt{2 \pi}\left(1-\epsilon^{2}\right)\left(k_{B} T\right)^{3 / 2} \\
& \times \sum_{i, j=1}^{2}\left(r_{i}+r_{j}\right)^{2}\left(\frac{m_{i}+m_{j}}{m_{i} m_{j}}\right)^{1 / 2} \int_{0}^{\infty} n_{i}(z) n_{j}(z) d z .
\end{aligned}
$$

The integral in the above expression is readily evaluated using Eqs. (5) and (6):

$$
\int_{0}^{\infty} n_{i}(z) n_{j}(z) d z=\frac{g}{\Omega^{2} k_{B} T} \frac{m_{i} m_{j}}{m_{i}+m_{j}} N_{i} N_{j}
$$

with which we finally obtain

$$
\begin{aligned}
Q= & \frac{4 \sqrt{\pi} g\left(1-\epsilon^{2}\right)}{\Omega} \sqrt{k_{B} T}\left(\sqrt{m_{1}} r_{1}^{2} N_{1}^{2}\right. \\
& \left.+\sqrt{m_{2}} r_{2}^{2} N_{2}^{2}+\sqrt{\frac{m_{12}}{2}}\left(r_{1}+r_{2}\right)^{2} N_{1} N_{2}\right) .
\end{aligned}
$$

Equating the two expressions for the rate of energy input [Eq. (A3)] and energy loss [Eq. (A11)] yields the granular temperature $T$ given in Eq. (8) in the main text.
[1] I. Goldhirsch and G. Zanetti, Phys. Rev. Lett. 70, 1619 (1993).

[2] H. M. Jaeger, S. R. Nagel, and R. P. Behringer, Rev. Mod. Phys. 68, 1259 (1996).

[3] A. Kudrolli, M. Wolpert, and J. P. Gollub, Phys. Rev. Lett. 78, 1383 (1997).
[4] L. Kadanoff, Rev. Mod. Phys. 71, 435 (1999).

[5] I. Goldhirsch, Annu. Rev. Fluid Mech. 35, 267 (2003).

[6] H. J. Schlichting and V. Nordmeier, MNU Math. Naturwiss. Unterr. 49, 323 (1996).

[7] J. Eggers, Phys. Rev. Lett. 83, 5322 (1999). 
[8] K. van der Weele, D. van der Meer, M. Versluis, and D. Lohse, Europhys. Lett. 53, 328 (2001).

[9] J. J. Brey, F. Moreno, R. García-Rojo, and M. J. RuizMontero, Phys. Rev. E 65, 011305 (2001).

[10] R. Mikkelsen, D. van der Meer, K. van der Weele, and D. Lohse, Phys. Rev. Lett. 89, 214301 (2002).

[11] J. L. Spradley, Am. J. Phys. 55, 183 (1987); J. S. Huebner and T. L. Smith, Phys. Teach. 30, 46 (1992).

[12] J. Schäfer, S. Dippel, and D. E. Wolf, J. Phys. I 6, 5 (1996).

[13] T. Schwager and T. Pöschel, Phys. Rev. E 57, 650 (1998).

[14] V. Kumaran, Phys. Rev. E 57, 5660 (1998).

[15] R. D. Wildman and D. J. Parker, Phys. Rev. Lett. 88, 064301 (2002).

[16] K. Feitosa and N. Menon, Phys. Rev. Lett. 88, 198301 (2002).

[17] V. Garzó and J. Dufty, Phys. Rev. E 60, 5706 (1999).

[18] W. Losert, D. G. W. Cooper, J. Delour, A. Kudrolli, and J. P. Gollub, Chaos 9, 682 (1999).

[19] A. Barrat and E. Trizac, Granular Matter 4, 57 (2002).

[20] M. Alam and S. Luding, Granular Matter 4, 139 (2002).

[21] For the highest parabolic flight in Figs. 3 and 5(c) the $z$ component of the velocity goes down from $v_{z}=2.03 \mathrm{~m} / \mathrm{s}$ at $z$ $=0.89 \mathrm{~m}$ to $0 \mathrm{~m} / \mathrm{s}$ at $z=1.10 \mathrm{~m}$, as the particle's vertical kinetic energy $\frac{1}{2} m_{2} v_{z}^{2}\left(=\frac{3}{2} T_{z}\right)$ is converted into potential energy $m_{2} g z$. The constant values of the other components, $v_{x}$ $=0.36 \mathrm{~m} / \mathrm{s}$ and $v_{y}=1.08 \mathrm{~m} / \mathrm{s}$, can be read off from Figs. 5(a) and 5(b) with $m_{2}=\frac{4}{3} \pi \rho r_{2}^{3}=0.063 \mathrm{~g}$.

[22] An alternative theoretical approach to the clustering phenomenon in granular gases is the so-called hydrodynamic model introduced by Brey et al in [9] and generalized to a bidisperse gas by Barrat and Trizac [19]. The two approaches are put side by side in K. van der Weele, R. Mikkelsen, D. van der Meer, and D. Lohse, in The Physics of Granular Media, edited by H. Hinrichsen and D. E. Wolf (Wiley, Berlin, 2004).

[23] The ideal-gas law normally applies for a gas with conservative (dissipationless) collisions and negligible excluded volume.
Both these conditions are met reasonably well in the present system: (a) The coefficient of restitution $\epsilon=0.85$ is not too different from 1, and (b) the gas is dilute enough to neglect excluded volume effects in first approximation. For more refined equations of state, see Refs. [24-26].

[24] J. T. Jenkins and M. W. Richman, Phys. Fluids 28, 3485 (1985).

[25] E. L. Grossman, T. Zhou, and E. Ben-Naim, Phys. Rev. E 55, 4200 (1997).

[26] S. Luding, Phys. Rev. E 63, 042201 (2001).

[27] J. J. Brey, M. J. Ruiz-Montero, and F. Moreno, Phys. Rev. E 62, 5339 (2000); 63, 061305 (2001).

[28] J. J. Brey and M. J. Ruiz-Montero, Phys. Rev. E 67, 021307 (2003).

[29] D. Paolotti, C. Cattuto, U. Marini Bettolo Marconi, and A. Puglisi, Granular Matter 5, 75 (2003).

[30] Naively integrating up to $h \rightarrow \infty$ would lead to an (unphysical) nonzero flux for $N_{i}=0$. To see this, consider a compartment without any type 2 particles: then $T \propto 1 / N_{1}^{2}$ [from Eqs. (8) and (9)] and hence $F_{1}$ loses its $N_{1}$ dependence in the dilute limit [cf. second line of Eq. (10)], making it nonzero for $N_{1}=0$.

[31] Because of a refinement in the flux model, the expressions for $K$ and $D$ differ slightly from the ones presented in [10]. Apart from a different scaling (in the parameter $D$ ) of the experiments onto the model, there are neither qualitative nor quantitative changes to the conclusions drawn in that paper.

[32] D. van der Meer, K. van der Weele, and D. Lohse, Phys. Rev. E 63, 061304 (2001).

[33] D. van der Meer, K. van der Weele, and D. Lohse, Phys. Rev. Lett. 88, 174302 (2002).

[34] Statistical fluctuations in the context of compartmentalized clustering have recently been discussed by several authors, see, e.g. [19] and U. Marini Bettolo Marconi and A. Puglisi, Phys. Rev. E 68, 031306 (2003). 